\title{
Introduction : à quoi servent les chiffres de la discrimination ?
}

\author{
VinCENT-ARnaud ChapPe \& MireILle EberhaRd
}

Vincent-Arnaud Chappe est sociologue, chargé de recherche au CNRS, membre du centre d'étude des mouvements sociaux à l'EHESS. Ses travaux portent sur les outils des politiques d'égalité dans le monde du travail.

vincent.arnaud.chappe@ehess.fr

Mireille Eberhard est maîtresse de conférences en sociologie (URMIS, Université de Paris). Ses recherches de terrain portent sur la mise en ouvre des politiques de lutte contre les discriminations, sur l'expérience du racisme et des discriminations, ainsi que sur les stratégies et réactions adoptées par les minoritaires. Elle a également travaillé sur la mesure de la perception et de l'expérience des discriminations.

mireille.eberhard@u-paris.fr

\section{Pour une sociologie de la quantification des discriminations}

Ce numéro des Cahiers de la LCD vise à interroger la question des appuis statistiques et de leurs effets sur la lutte contre les discriminations. Dans le contexte français, la simple évocation de la quantification des discriminations n'est pas sans soulever d'âpres débats récurrents et bien connus, notamment s'agissant des discriminations ethnoraciales. Depuis la «controverse des démographes » de la fin des années 1990, les polémiques se succèdent et ressurgissent à rythme régulier, prenant un tournant plus directement politique avec l'introduction de la lutte contre les discriminations sur l'agenda au cours des années 2000. Alors qu'elles étaient plutôt cantonnées à une sphère restreinte et académique, les controverses autour desdites «statistiques ethniques » ont été fortement médiatisées et ont impliqué davantage d'acteurs (institutionnels, politiques, associatifs) durant la présidence de Sarkozy et, notamment, à la suite du projet de révision de la loi Informatique et liberté de 1978 et de la décision du Conseil constitutionnel qui s'est ensuivie. Les (op)positions apparaissent figées entre les partisans d'une facilitation de la production et de l'usage de statistiques pour dévoiler et lutter contre les discriminations raciales et ceux pour qui une telle production fige et, par là même, enferme les individus et les groupes dans des identifications réifiées contraires aux principes républicains. Pour les uns, la statistique permet de dévoiler une vue d'ensemble, un ordre de grandeur que ne permet pas la seule approche qualitative et en ce sens, permet de « rendre visible l'invisible » (Simon, 2020), cette factualisation des discriminations étant un préalable nécessaire à une intervention visant la transformation des mécanismes systémiques qui les produisent. Pour les autres, les identifications sont labiles et fluctuantes et l'acte de classement sur lequel repose le dénombrement statistique publicise, institutionnalise et, ce faisant, participe à la reproduction et à la consolidation d'identités prescrites ethnicisantes et racisantes qu'il faut justement combattre (CARSED, 2009).

À côté des controverses entourant la catégorisation des groupes «à risques » potentiellement exposés au phénomène, les débats scientifiques relatifs à la quantification des discriminations portent aussi sur les questions de méthodes, et des apports et limites propres à chacune d'elles. Les enquêtes relatives à la mesure des discriminations se sont multipliées et ont connu des développements méthodologiques constants. Trois approches complémentaires peuvent être distinguées (Delattre et al., 2014). La première approche consiste à mettre en évidence des différences objectives entre différents groupes (différences de position, différences de situation). Dans une démarche " toute chose égale par ailleurs », cette démarche analytique s'attache à mettre en évidence un "résidu discriminatoire », associé à l'effet propre de la caractéristique étudiée, une fois pris en compte les effets d'autres variables incluses dans un modèle statistique. Si cette approche permet de mettre en 
évidence des inégalités de situation au niveau global, elle ne permet pas vraiment de saisir les processus et mécanismes qui participent de leur (re)production. L'interprétation des écarts inexpliqués en termes de discrimination est également sujette à discussion (Jugnot, 2019 ; Delattre et al., 2014). En effet, la part inexpliquée des inégalités dépend du nombre et du degré de détail des variables explicatives retenues dans le modèle statistique (et, corrélativement, disponibles dans les données d'enquête). La robustesse de ce type d'analyse est donc doublement conditionnée par la qualité des informations mobilisables et par le nombre d'observations disponibles.

La seconde approche renvoie à la mise en place de tests de situation, ou testing. Expérimental - au sens d'" expérience scientifique » grandeur nature -, ce type d'enquêtes consiste à tester des candidatures en envoyant des propositions fictives, par paire, en ne faisant varier que la caractéristique testée. Les différences de traitement entre les différentes paires sont alors considérées comme discriminatoires (Cédiey et al., 2007). Ces dernières années, ces études se sont multipliées, tant du point de vue des critères testés que du point de vue des champs étudiés. Ces travaux restent toutefois cantonnés à des situations localisées et leur validité reste limitée au métier, à la zone et à la période testés sans pouvoir être généralisée à d'autres contextes (Petit et al., 2020 ; L'Horty et al. 2019).

La dernière approche vise à enregistrer l'expérience et la perception des discriminations, telle que rapportée par les enquêtés. Cette approche mobilise plusieurs types d'indicateurs. Les premiers, construits à partir de questions d'opinion, portent sur les représentations à l'égard des discriminations et évaluent la propension à penser que les discriminations peuvent advenir. Les seconds portent sur l'expérience auto-reportée des discriminations (déclarer penser avoir subi des traitements inégalitaires ou des discriminations). Les troisièmes renvoient à des « discriminations situationnelles » en tant qu'ils se réfèrent à la description de situations concrètes et contextualisées (Simon et Lesné, 2012). Dans cette troisième approche, la mesure porte sur l'appréciation que les enquêtés ont des discriminations ou des traitements injustes qu'ils déclarent avoir vécus. La dimension subjective de ces différents indicateurs peut être sujette à discussions car s'ils mettent au jour la prévalence d'expérience relevant de la discrimination, ils reposent également sur la capacité que les enquêtés ont de reconnaître, d'interpréter et, finalement de déclarer une expérience vécue comme telle dans le cadre d'un questionnaire fermé. Cette propension dépend elle-même de facteurs sociaux objectifs (sexe, position sociale, capital scolaire), mais également d'éléments subjectifs (processus d'auto et d'hétéro-identification à un groupe minoritaire, estime de soi) qui la conditionnent (Primon, 2014).

Si le débat est largement entamé sur la question de la mesure des discriminations et de l'entrecroisement des questions scientifiques, éthiques et politiques qui rentrent en jeu, nous souhaitons dans ce numéro élargir la réflexion. Les analyses sur la quantification des discriminations se sont en effet largement concentrées sur un espace spécifique qui est celui des sciences sociales dans ses différentes composantes (sociologie, économie, démographie, etc.). Les controverses sur l'objectivation des discriminations ou sur la pertinence des catégories ethnoraciales ont d'abord concerné les chercheurs universitaires et les grandes bases de données statistiques sur lesquelles ils peuvent s'appuyer pour objectiver la réalité. Bien sûr, la dynamique du débat (et sa violence) a eu pour conséquence un important décloisonnement des prises de position et des arènes de discussion : nombreuses sont ou ont été les interventions politiques et/ou médiatiques où est discutée la question de la pertinence de la mesure des discriminations. Mais il nous semble néanmoins que cette ouverture de l'espace de prise de position n'a pas fondamentalement fait évoluer le centre de gravité du débat : il s'agit toujours de réfléchir et se positionner sur la pertinence des catégorisations et des mesures dans la statistique publique - les données du recensement ou les grandes enquêtes ad hoc d'institutions publiques comme l'INSEE ou l'INED - mais sans prendre en considération d'autres espaces, éventuellement moins centraux ou visibles, où s'observent également des pratiques et controverses statistiques en matière de discrimination. D'autant que si les débats ont essentiellement porté sur les statistiques dites ethniques, la quantification des discriminations concerne également d'autre critère, notamment celui du sexe. 
À cet égard, notre ambition est double. Il s'agit tout d'abord d'élargir le périmètre des espaces pris en compte : la question statistique ne se donne pas uniquement à voir à un niveau «macro », entendu notamment au niveau national. Elle se pose également à des niveaux plus locaux, dans les entreprises, les collectivités territoriales, les arènes judiciaires, les espaces militants, etc. Mais il s'agit également d'élargir le questionnement concernant les «pratiques » de la statistique des discriminations. On a vu que la majorité des débats scientifiques porte à la fois sur la question de la pertinence (scientifique ou politique) des catégories d'identification des groupes potentiellement discriminés et sur celle de la «calculabilité » des discriminations. La question de la circulation et de l'usage des données statistiques de discrimination produites reste néanmoins largement en retrait : des réflexions existent sur le potentiel des statistiques genrées (Blanchard et Pochic, 2021) ou raciales (StavoDebauge, 2003), mais les mécanismes effectifs (ou attendus) d'une performativité de la quantification n'ont pas fait l'objectif d'une explication et d'une comparaison systématique. L'objectif de ce dossier est donc de poser encore plus explicitement la question des conséquences de la mesure des discriminations (tout critère confondu), tout en réinscrivant cet intérêt dans la prise en compte de l'ensemble de la trajectoire des chiffres de la discrimination dans l'espace social.

Pour ce faire, nous pouvons nous appuyer sur le corpus grandissant en sociologie de la quantification, domaine d'intérêt majeur des sciences sociales depuis une trentaine d'années (Martin, 2020). Si les études de quantification ont d'abord porté sur la question du raisonnement statistique et des conventions d'équivalence et de commensurabilité qui permettent la mise en chiffre du réel (Espeland, 1998), de nombreux travaux ont depuis exploré la question de l'usage des chiffres, qu'il soit le fait de l'État (Desrosières, 2000 ; Didier, 2009) ou de divers acteurs professionnels (Porter, 1996). Des recherches récentes portent sur l'hégémonie actuelle des chiffres dans la gouvernance de société à travers le développement toujours croissant d'indicateurs et de pratiques de benchmarking (Bruno et Didier, 2013 ; Bezès et al., 2016 ; Supiot, 2015), phénomène également massif dans la sphère du travail (Chauvel et Pillon, 2020). Un nouveau champ de recherche autour du « statactivisme » (contraction de statistiques et d'activisme) analyse également la question de l'usage militant des chiffres pour contester les représentations de la réalité produites par les statistiques institutionnelles (Bruno et al., 2014).

Précisons également que nous avons à cœur de montrer que si les chiffres ont une « carrière » au sens sociologique du terme, celle-ci ne doit pas être comprise comme une trajectoire linéaire associant de façon étroite production, usage et effets. À la manière de la balistique des arguments théorisée par Francis Chateauraynaud (2011), les données statistiques produites sont susceptibles d'échapper à leurs créateurs, d'avoir des usages ou des effets non anticipés, voire contradictoires au regard de leurs finalités escomptées. Il s'agit ainsi de penser de façon complexe les agencements, articulations et tensions entre différents espaces qu'on peut distinguer de façon analytique : des espaces d'élaboration méthodologique où se construisent des théories et des dispositifs de mesure des discriminations ; des espaces de production des chiffres; des espaces d'usage, où les chiffres sont utilisés par des acteurs dans le cadre d'un agir instrumental, susceptible d'obéir à différentes logiques (car les chiffres n'agissent pas d'eux-mêmes mais à travers des médiations qui participent de leur pouvoir) ; des espaces de réception qui permettent de poser la question de ce que font les chiffres et des réactions, plus ou moins directes, qu'ils engendrent (Espeland et Sauder, 2007).

À titre d'illustration, nous souhaitons ouvrir ici la réflexion en présentant - de façon non exhaustive différentes logiques d'usage des chiffres de discrimination, puis en présentant des situations empiriques où les chiffres de discrimination ont circulé dans des espaces différents.

\section{Logiques plurielles des chiffres de la discrimination et circulation entre différents espaces sociaux}


Nous souhaitons distinguer ici différentes logiques associées aux chiffres de la discrimination. Par logique, nous postulons ici une situation de cohérence idéal-typique entre les modalités de production des chiffres, leurs usages et leurs effets. Cette logique implique ainsi des configurations réunissant acteurs, modes de preuve et dispositifs de réception spécifiques.

Dans un second temps, nous reviendrons sur cette notion de logique à partir de deux exemples qui viendront illustrer la manière dont les chiffres circulent entre des espaces et mondes sociaux hétérogènes, ce qui n'est pas sans provoquer de tensions.

\subsection{La logique scientifique}

La première logique des statistiques de discrimination, la plus évidente peut-être (du point de vue de chercheur·e's en tout cas), est de faire connaissance. Cette logique scientifique est d'abord liée au travail de professionnels de la science, au sens d'individus disposant de compétences spécifiques et institutionnalisées dans l'objectivation du réel. Concernant la question des discriminations, elle implique des chercheur·e's en sciences sociales (sociologues, économistes, gestionnaires, etc.) qui disposent de la maîtrise de méthodes rigoureuses, plus ou moins routinisées, pour mesurer la discrimination (régressions, testing, analyse de variance, etc.) ou sa perception. Ces méthodes ont fait l'objet d'une élaboration réflexive au sein des communautés scientifiques concernant leur pertinence, leur signification et leur mise en œuvre (v. par ex., pour les sciences économiques, Chassonnery-Zaïgouche, 2013).

La logique scientifique implique en premier lieu que la mesure des discriminations soit produite de la communauté scientifique vers la communauté scientifique : l'activité de production scientifique n'est pas une activité solitaire mais inscrite dans des collectifs, parfois informels, mais souvent institutionnels (équipes de recherche financées, laboratoires, réseau ou association scientifique, etc.). Les résultats de la recherche sont discutés dans des séminaires et des colloques, et évalués dans l'optique de publications, d'évolution de carrière ou de nouveaux financements.

Dans cette logique scientifique, la production de statistiques sur la discrimination sert d'abord à l'accumulation de connaissances sur la réalité du monde social, que ce soit à des fins descriptives (rassembler des éléments empiriques) ou explicatives et théoriques (produire des concepts et éclairer des processus ou causalités permettant de faire sens de ce qui est observé). Bien sûr, cette logique scientifique ne se suffit que rarement à elle-même, comme nous le montre d'ailleurs dans ce numéro l'entretien avec Dominique Meurs, et de nombreux chercheur.e.s encouragent ou reconnaissent, de façon directe ou indirecte, des usages extrascientifiques de leurs résultats empiriques. À cet égard, le champ d'étude des discriminations est assez faiblement autonome par rapport au champ social et politique. Les politiques publiques ont largement participé à l'institutionnalisation de ce domaine d'étude scientifique, ce qui n'est pas sans incidence sur la circulation de chiffres scientifiques hors de leur espace de production . Mais, au moins de manière idéal-typique, ceux-ci sont dépendants des conditions d'élaboration, de réception et de validation propres au champ scientifique.

\subsection{La logique probatoire}

Une deuxième logique identifiable est celle qui vise à faire preuve, au sens judiciaire du terme : la logique probatoire concerne les cas où la production d'un chiffre cherche à produire une saillance empirique, au service d'un énoncé sur la réalité, dans le cadre d'une épreuve conflictuelle. Cette logique n'est pas directement celle de la preuve scientifique, dans laquelle les acteurs sont censés partager un but commun dans l'élucidation du réel. Il s'agit ici de caractériser des situations qui, sur le modèle du contentieux judiciaire, voient s'opposer des parties cherchant à faire reconnaître leur propre interprétation de la réalité.

Dans cette logique, c'est la volonté de faire reconnaître une situation comme discriminatoire qui motive la production du chiffre. Dans les procès en matière de discrimination par exemple, si la preuve statistique est souvent centrale comme nous l'explique Robin Médard Inghilterra dans ce numéro, elle n'est pas forcément première dans le contentieux : la conviction pour les parties dénonciatrices d'avoir à faire des discriminations (conviction qui peut par exemple être préalablement fondée sur une accumulation de témoignages individuels) peut amener les acteurs à vouloir produire une quantification qui vient confirmer leur interprétation des faits. 
Cela ne veut pas dire que les chiffres ainsi produits sont des «faux » servant uniquement à confirmer une définition préalable de la situation, mais qu'ils sont d'abord produits dans une optique de confirmation - et de factualisation - d'une réalité que la partie accusatrice sait être véritable. A contrario, dans le cadre d'une procédure contradictoire, la production d'autres chiffres par les parties mises en cause ne vise pas tant à produire une vérité, au sens scientifique du terme, qu'à remettre en cause le discours et l'argument porté par la partie adverse.

Précisons que dans cette logique où la production des chiffres est seconde par rapport à l'argument, elle n'est pas spécifiquement le fait de professionnels de la science. Elle est souvent mise en œuvre et portée directement par les parties prenantes du conflit, et se déploie ainsi dans les objets et arènes qui y sont liées (conclusions des parties, plaidoiries des avocats). Dans le cadre d'un procès, les chiffres sont ainsi souvent construits par les plaignants ou ceux qui les assistent (avocats, syndicalistes, militants associatifs, etc.) et obéissent donc à des logiques de production externes à la logique proprement scientifique. Leur élaboration s'inscrit dans des conflits de qualification qui visent à homologuer ou à invalider une situation comme relevant de la discrimination et à emporter la conviction de celles et ceux en position de pouvoir départager les parties (les juges dans le procès). Cet enjeu nécessite que puisse être prise en compte la solidité apparente de la méthodologie sur laquelle l'opération de mesure repose. Toutefois, l'appréciation de cette solidité ne repose pas forcément sur les mêmes bases que l'évaluation de la rigueur du protocole méthodologique opérée dans les arènes de discussion scientifique (Leclerc, 2013). D'autant que la réception de ces formes de quantification dépend également des règles de fonctionnement intrinsèques à l'épreuve qui obéissent à d'autres logiques que celles des arènes scientifiques : dans le cas des procès judiciaires, des règles de droit façonnent ainsi la légitimité et l'acceptabilité de certaines preuves de discrimination.

\subsection{La logique de conscientisation}

Une troisième logique vise à faire prendre conscience de la discrimination à partir de son objectivation chiffrée. Cette logique de conscientisation est plurielle : elle englobe un ensemble de phénomènes par lesquels une situation fait l'objet d'une prise de conscience de la part d'un acteur ou d'un groupe. Dans le cas de la discrimination, le phénomène peut en effet soit ne pas être reconnu ou qualifié comme tel, soit être intériorisé, au point de le considérer comme normal et, par là même, ne susciter aucune forme de réaction ou de prise en compte spécifique. Dans ce sens, la conscientisation par le chiffre n'est pas qu'une question de savoir pur : elle repose sur une capacité de décryptage et de qualification qui implique un processus de scandalisation morale (prendre conscience de l'existence et l'ampleur d'un fait moralement inapproprié et par là même le dénoncer) et de concernement (se sentir, directement ou indirectement, heurté par l'indignité morale et l'injustice ainsi dévoilées).

Cette logique de conscientisation se rapproche d'une pratique de dévoilement revendiquée par la sociologie critique : il s'agit, à travers la factualisation des chiffres, de montrer en quoi la réalité s'éloigne d'un discours mythologique revendiqué par les institutions - par exemple celui de l'égalité républicaine ou de la méritocratie. Elle peut être portée par des acteurs militants, ou par des institutions qui, à l'instar du Défenseur des droits ou de la Commission nationale consultative des droits de l'Homme, sont chargés (entre autres) d'inciter les acteurs publics à la prise en charge de la question des discriminations. Les chiffres produits dans ce cadre sont publiés dans des rapports et des communiqués qui ont vocation à circuler dans l'espace public et médiatique.

Ce voile levé sur la réalité, en activant le ressort de l'injustice, est pensé comme une condition de possibilité des mobilisations (individuelles ou collectives) contre la discrimination. Ce type de logique peut être rapproché de la notion de «statactivisme ", la production de chiffre relevant d'une démarche politique visant à faciliter l'émergence et la mobilisation d'un public concerné. L'effet de conscientisation par les chiffres peut être recherché directement - comme dans le cas des «statistiques populaires » du CRAN présenté par Jawad Bouadjaja - ou être plus indirectement lié à la circulation large de données dans l'espace public et médiatique, ce que montrent les entretiens réalisés par Élodie Druez auprès de diplômé·e $\cdot s$ d'origine subsaharienne au Royaume-Uni en comparaison avec le cas. 
Ici encore, la force de ces chiffres est moins caractérisée par la solidité de leur construction en soi, que par leur lisibilité et leur transportabilité : pour être susceptibles de produire cet effet de conscientisation, les chiffres doivent pouvoir circuler et être appropriés facilement. La compréhension de leur signification doit être aisée, quitte à mettre à distance le souci de la rigueur statistique .

\subsection{La logique de diagnostic}

Une troisième logique vise à faire diagnostic : par diagnostic, on entend que ces chiffres peuvent servir à alimenter la réflexivité d'une entité en tant que celle-ci relève d'un espace social identifiable (que ce soit une organisation, une entreprise, une administration, un territoire, etc.). En produisant un diagnostic, il s'agit ainsi pour l'acteur qui en est à l'initiative d'objectiver la discrimination susceptible de s'inscrire dans l'espace dont il est responsable.

On peut relever des formes très différentes du diagnostic : celui-ci peut être ponctuel ou régulier, voire continu comme dans la pratique du monitoring ; ciblé ou multidimensionnel ; s'inscrire dans une démarche d'évaluation de pratiques identifiées ou au contraire être préalable à toute entreprise de réforme organisationnelle ou territoriale ; relever ou non d'une obligation légale - comme dans le cas du rapport de situation comparée entre femmes et hommes dans l'entreprise étudiée par Vincent-Arnaud Chappe et Hélène Demilly -, ou être nécessaire à l'obtention d'un label, comme dans le cas du label de la diversité pour les collectivités territoriales (Bereni et al., 2020) ; ne chercher que l'objectivation interne des discriminations ou s'inscrire dans une démarche de comparaison par rapport à d'autres entités comme dans le cas du benchmarking (Blanchard, 2018). Ces diagnostics peuvent être réalisés par des acteurs internes à l'organisation, ce qui peut les conduire à devoir s'approprier des outils d'objectivation ne relevant pas forcément de leur champ de compétence. Ils peuvent également être l'occasion de faire intervenir la figure de l'expert (Cédiey et Maillet, 2010), son savoir spécialisé étant dans ce cas-là mis à disposition des objectifs de l'organisation, en contrepartie d'une rémunération ou d'un accès à des données valorisables dans l'espace scientifique (Guillaume et Pochic, 2011 ; v. aussi l'entretien avec Dominique Meurs dans ce numéro). Le plus souvent, ces chiffres font l'objet de pratiques de communication interne, notamment sous forme de rapports. Toutefois, ils peuvent également donner lieu à des pratiques d'affichage à destination de publics plus ou moins larges.

Ce qui nous semble être caractéristique de la logique de diagnostic est que les chiffres produits doivent, en théorie, servir à éclairer et orienter l'action - et ce même si dans bien des cas les diagnostics se suffisent à euxmêmes et jouent principalement un rôle «symbolique » en étant déconnectés de toute réforme substantielle de l'organisation (Ahmed, 2007). Dans une perspective idéal-typique, la quantification des discriminations est ici directement liée à une perspective pragmatique, au sens où elle doit servir l'action - que ce soit en la préparant en amont ou en la réorientant. Ce lien à l'action peut, certes, être plus ou moins immédiat : le diagnostic peut n'être qu'exploratoire, sans certitude que sa réalisation débouche effectivement sur des pratiques concrètes. Ces dernières relèvent néanmoins toujours (dans notre typologie) de son horizon d'attente. Cette visée implique également, en théorie, une certaine transparence interprétative des résultats leur permettant d'accéder au statut d'« indicateurs prégnants » (Boussard, 2001) susceptibles d'orienter les décisions (par opposition à une ambiguïté interprétative susceptible de paralyser toute velléité réformatrice).

\subsection{La logique d'obligation}

La dernière logique repérée est celle de faire obligation : il s'agit ainsi de désigner des pratiques de quantification qui portent en elle une normativité contraignante. Contrairement à la logique de diagnostic où les chiffres ouvrent vers l'action sans la déterminer, la logique d'obligation revêt une causalité beaucoup plus directe.

Typiquement, les politiques de quota relèvent de cette logique : elles impliquent une quantification du réel (ratio femmes-hommes sur une liste syndicale ou électorale ou dans un conseil d'administration, pourcentage de travailleurs en situation de handicap parmi les employés, pourcentage de boursiers, etc.) et une comparaison avec une norme fixée par le droit. L'existence d'un écart est en effet susceptible d'enclencher une sanction de l'acteur contrevenant (amende, invalidation du fait mis en cause, etc.). Le caractère contraignant peut être décalé dans le temps, comme dans le cas de l'index d'égalité professionnelle où les entreprises ayant une note inférieure au 
seuil couperet de 75 ont 3 ans pour redresser la barre sous peine d'une sanction financière. Il peut prendre également des formes plus souples comme dans le cas de l'Equal Employement Opportunity Commission (EEOC) aux États-Unis : les entreprises de plus de 25 salariés ont dans l'obligation de fournir à l'EEOC des données chiffrées sur la composition raciale et sexuée de leur main-d'œuvre, qui sont comparées aux chiffres équivalents du bassin d'emploi. L'EEOC a alors la possibilité d'engager une enquête - voire un contentieux - en cas d'écart trop important entre ces deux niveaux de mesure. De la même façon, dans le cas d'écarts statistiques significatifs, les entreprises contractant avec l'administration fédérale sont dans l'obligation de mettre en place des mesures proactives visant à rééquilibrer leur main-d'œuvre.

La spécificité de cette logique est donc d'adosser systématiquement la mesure du réel à une quantité théorique socialement désirable (par exemple, la parité à hauteur de $40 \%$ pour les conseils d'administration des moyennes et grandes entreprises françaises). Par rapport à la logique de diagnostic qui nécessite déjà une quantification facilement « activable », la logique d'obligation va encore plus loin, en exigeant la production de chiffres parfaitement normés dans leur construction et ne souffrant d'aucune marge d'interprétation. Elle est donc fortement liée à un travail de définition administrative concernant la procédure de quantification à suivre. Dans cette optique, la production chiffrée ne peut qu'être simple et réduite à un nombre très limité d'indicateurs - souvent un seul - afin de permettre l'opération de comparaison avec la norme fixée. La logique d'obligation implique ainsi un travail de réduction de la pluri dimensionnalité des formes de la discrimination dans une représentation synthétique de la réalité et des objectifs à atteindre.

La définition extrêmement normée des indicateurs retenus permet en général la délégation de leur production à des acteurs non spécialisés ou à des logiciels automatisés. Ces chiffres, s'ils peuvent faire l'objet de pratiques de communication plus ou moins larges en fonction de la réalité qu'ils donnent à voir, sont en priorité amenés à transiter vers des dispositifs administratifs où ils seront mis en comparaison avec les normes d'égalité qui s'imposent aux organisations.

\subsection{Des logiques enchevêtrées}

La présentation que nous avons faite des différentes logiques de quantification de la discrimination est idéaltypique. Dans la réalité telle qu'elle s'observe à travers l'enquête, les raisons de produire des chiffres sont multiples, les frontières entre les acteurs et groupes professionnels sont poreuses, les outils de calcul et leurs résultats circulent entre différents dispositifs espaces sociaux. Les logiques scientifiques de production de connaissance peuvent ainsi très bien s'agencer avec des visées en termes de conscientisation ou de politisation, tout comme les chercheurs peuvent être mis à contribution dans le cadre de démonstrations probatoires ou sollicités par des acteurs professionnels pour les aider à réaliser un diagnostic. De la même façon, des logiques de diagnostic et d'obligation peuvent très bien s'entrecroiser, les chiffres pouvant servir à la fois à orienter l'action à venir tout en faisant peser une contrainte normative forte sur l'organisation quantifiée. Deux exemples empiriques viennent illustrer cet entrecroisement des logiques.

Le premier a trait à la question des contrôles d'identité discriminatoires de la police, dits « contrôles au faciès » (Boutros, 2020). En 2007, l'Open Society Justice Initiative finance une étude sur la question. Menée par Fabien Jobard et René Lévy - tous deux chercheurs au CNRS et membres du laboratoire CESDIP - à partir d'observations quantifiées des contrôles d'identité effectués sur cinq sites parisiens, la recherche conclut à la surreprésentation des minorités visibles parmi les populations contrôlées. Si l'étude obéit aux standards scientifiques et est à ce titre publiée dans des revues spécialisées (Jobard et al., 2012), elle fait l'objet d'une importante communication politique par l'Open Society Justice Initiative qui vise à faire émerger le problème dans les arènes publiques. Ces chiffres ont enfin été utilisés dans le cadre d'un procès qui a opposé à partir de 201213 plaignants à l'État français, aboutissant à sa condamnation définitive pour faute lourde par la Cour de cassation en 2016 pour cinq de ces 13 cas : sans pouvoir directement prétendre au statut de preuve, ces données ont servi dans l'argumentation à montrer le caractère répandu des discriminations alléguées par les plaignants individuels. 
Un autre exemple de cet enchevêtrement des logiques est celui du testing : utilisé en France depuis le début des années 2000 à la fois comme mode de preuve scientifique et judiciaire (Calvès, 2007), il devient également un instrument d'action publique saisi par les gouvernements à diverses reprises. En 2018, à la suite de l'engagement préalable du candidat Macron, le gouvernement d'Édouard Philippe charge une équipe d'économistes de procéder à des testing de grande ampleur : 103 grandes entreprises françaises, sur six régions, sont testées. L'envoi de 17163 candidatures montre que les candidats ayant un CV comportant un nom et prénom identifié comme maghrébin sont moins souvent contactés que les candidats de référence (Challe et al., 2020). Là encore, cette enquête obéit à la fois à une logique scientifique - pour les économistes, mieux comprendre le phénomène des discriminations à partir notamment d'une approche « multicanal " - mais également diagnostic : pour le gouvernement, il s'agit de faire un état des lieux des discriminations à l'emploi dans la perspective du déploiement d'une politique publique de discrimination (la pratique devant d'ailleurs être reconduite dans l'avenir). Mais les résultats statistiques obtenus à partir de l'agrégation des résultats vont également faire l'objet d'une action plus ciblée : mis sous pression par la société civile, le gouvernement communique le nom de six entreprises épinglées comme discriminantes - dans une logique de name and shameet annonce qu'elles seront obligées de suivre une formation au recrutement non-discriminant. Le dévoilement de l'identité des entreprises entraîne alors de vives protestations de la part du MEDEF, qui met en cause publiquement la méthodologie scientifique déployée dans le cadre du testing. Tandis que l'exemple précédent montrait un agencement harmonieux entre des logiques différentes, celui-ci dévoile au contraire des tensions entre une logique scientifique, une logique de diagnostic et une logique d'obligation dans le cadre du déploiement de politiques de lutte contre les discriminations au travail.

La présentation de ces cinq idéaux-types mériterait de plus amples développements, concernant les acteurs qui les portent, les obstacles qu'ils rencontrent, les différents agencements possibles entre logiques de quantification, les potentialités de ces croisements mais également les tensions qu'ils peuvent faire émerger. Loin de vouloir clore le débat, l'objectif de cette introduction et de ce numéro est au contraire d'ouvrir un espace de recherche et de réflexion sur ce que peuvent faire les mesures de la discrimination, au-delà des batailles rangées entre «pro » et « anti » statistiques ethniques. S'intéresser aux différents contextes de production des chiffres et de la pluralité de leur usage peut ainsi permettre de renouveler à nouveaux frais un débat scientifique et politique, en insistant sur les potentialités - mais aussi les limites - de ces opérations de quantification.

\section{Présentation du numéro}

Les articles qui suivent nous présentent des perspectives variées sur les questions qui animent ce numéro : les deux premiers portent principalement sur l'amont de la chaîne de production des chiffres, mais dans des contextes extrêmement différents. L'article de Jawad Bouadjaja raconte ainsi comment le Conseil représentatif des associations noires de France (CRAN) a voulu encourager la production de «statistiques populaires » sur les discriminations. L'auteur nous détaille les attendus de cette action en termes de conscientisation, ainsi que les opérations cognitives et pratiques qu'il a lui-même appliquées dans le cadre d'une opération de comptage réalisée dans le cadre de son terrain ethnographique.

L'entretien réalisé avec Dominique Meurs permet de revenir sur les conditions et obstacles relatifs à la production de résultats quantifiés sur les discriminations, et notamment sur la question de l'accès aux données et variables pertinentes. Dominique Meurs aborde également les différents usages organisationnels et diagnostics qu'ont pu avoir ses recherches, que ce soit dans le cadre de collaborations avec des organisations publiques ou des entreprises.

Les deux articles suivants portent plus directement sur les usages de la quantification des inégalités et discriminations. L'article de Vincent-Arnaud Chappe et Hélène Demilly analyse ainsi les modalités de production concrètes et les limites du rapport annuel sexué que les entreprises sont dans l'obligation de produire sur les situations d'emploi. Il donne à voir la manière dont ce rapport, qui doit servir de base informationnelle aux politiques d'entreprise d'égalité entre femmes et hommes, est largement cadré par les choix de l'équipe de 
direction de l'entreprise. Il montre également comment, dans certaines situations, ces diagnostics chiffrés peuvent servir d'appui à l'ouverture de débats plus ou moins conflictuels entre directions d'entreprise et syndicats.

L'entretien réalisé avec Robin Médard vient éclairer une autre logique de quantification : il nous montre à quel point l'argument statistique est central dans la preuve judiciaire des discriminations, mais également comment l'accès aux données sur lesquelles repose la comparaison statistique est potentiellement problématique pour les plaignants. Robin Médard nous explique également les modes d'affinité entre la preuve statistique et les avancées les plus récentes du droit de la non-discrimination permettent de sortir d'une conception purement intentionnelle et curative de la discrimination.

Le dernier article traite des effets plus indirects de la quantification des discriminations. Élodie Druez s'appuie sur une approche comparée de l'expérience du racisme et des discriminations entre diplômés d'origine subsaharienne français et britanniques. Elle montre comment, dans ce deuxième cas, la présence importante de statistiques ethniques dans les espaces publics et médiatiques permet aux concernés de construire un discours sur le caractère systémique et institutionnel de la discrimination. Dans le cas français mis en contraste, l'illégitimité de ces formes de quantification empêche en revanche ce travail de montée en généralité, les discriminations étant à l'inverse principalement appréhendées à partir d'un cadre interindividuel et dépolitisé.

L'ensemble du dossier se propose ainsi d'éclairer de manière empirique, sous plusieurs angles, à différentes échelles et dans des contextes variés, les modalités de production, d'usage et d'appropriation des chiffres de la discrimination ainsi que leurs effets et leurs limites.

\section{Bibliographie}

Ahmed S. (2007), " "You end up doing the document rather than doing the doing" : Diversity, race equality and the politics of documentation », Ethnic and racial studies, vol. 30, n 4, pp. 590609.

Bereni L., Epstein R., Torres M. (2020), « Colour-blind diversity : how the "Diversity Label" reshaped antidiscrimination policies in three French local governments », Ethnic and Racial Studies, vol. 43, $\mathrm{n}^{\circ} 11$, pp. 119.

Bezès P., Chiapello E., Desmarez P. (2016), «Introduction : la tension savoirs-pouvoirs à l'épreuve du gouvernement par les indicateurs de performance ». Sociologie du travail, vol. 58, n 4, pp. 34769.

Blanchard S. (2018), « Le benchmarking au service de l'égalité ? » Gouvernement et action publique, vol. 4, n 4, pp. 3961.

Blanchard S. et Pochic S. (2021), Quantifier l'égalité au travail. Outils politiques et enjeux scientifiques, Presses universitaires de Rennes.

Boussard V. (2001), «Quand les règles s'incarnent L'exemple des indicateurs prégnants ». Sociologie du Travail, vol. $43, \mathrm{n}^{\circ} 4$, pp. 53351.

Boutros M. (2020), «Les mobilisations à l'épreuve de l'opacité policière en France », Lien social et Politiques, $\mathrm{n}^{\circ} 84$, pp. 4262.

Bruno I., Didier E. et Prévieux J. (2014), Statactivisme. Comment lutter avec des nombres, La Découverte.

Calvès G. (2007), «Au service de la connaissance et du droit : le testing », Horizons stratégiques, $n^{\circ}$ 3, pp. 816.

CARSED (Commission alternative de réflexion sur les «statistiques ethniques » et les discriminations) (2009), Le retour de la race - Contre les « statistiques ethniques », Éditions de l’Aube.

Cédiey É., Desprès C., L’Horty Y. (2007), « Le testing scientifique, problèmes de méthode », Horizons stratégiques, $\mathrm{n}^{\circ} 5$, pp. 75-91.

Cédiey É., Maillet E. (2010), « Sciences sociales et lutte contre la discrimination en entreprise : le cas d'Adecco », Tracés. Revue de Sciences humaines, ${ }^{\circ}$ 10, pp. 7782.

Challe L., Chareyron S., L'Horty Y. et Petit P. (2020), " Discrimination dans le recrutement des grandes entreprises : une approche multicanal », document de travail [en ligne].

Chassonnery-Zaïgouche C. (2013), «L'économie des discriminations : conquête impérialiste ou contribution à la théorie des prix ?», Economia. History, Methodology, Philosophy, n 3, vol. 1, pp. 87118. 
Chateauraynaud F. (2011), Argumenter dans un champ de forces : Essai de balistique sociologique, Éditions Pétra.

Chauvel S., Pillon J.-M. (2020), « Avant-propos », Sociologies pratiques, vol. 40, n 1, pp. 110.

Delattre E., Leandri N., Meurs D. et Rathelot R. (2013), « Introduction - Trois approches de la discrimination : évaluations indirectes, expérimentation, discriminations ressenties », Économie et Statistique, dossier « Inégalités et discriminations : questions de mesure », pp. 464-465-466.

Desrosières A. (2000), La politique des grands nombres : histoire de la raison statistique, La Découverte.

Didier E. (2009), En quoi consiste l'Amérique ? Les statistiques, le New Deal et la démocratie, La Découverte.

Espeland N. W. et Sauder M. (2007), «Rankings and Reactivity : How Public Measures Recreate Social Worlds $1 »$, American journal of sociology, vol. 113, $\mathrm{n}^{\circ} 1$, pp. 140.

Espeland W. N. et Stevens M. L. (1998), « Commensuration as a Social Process », Annual Review of Sociology, $\mathrm{n}^{\circ} 24$, pp. 313-343.

Guillaume C. et Pochic S. (2011), «Peut-on enquêter sur l'égalité professionnelle sans intervenir ? Retour sur une recherche en entreprise » in Des sociologues sans qualités ? Pratiques de recherche et engagements (Naudier D., dir.), La Découverte, pp. 11733.

Jobard F., Lévy R., Lamberth J. et Névanen S. (2012), « Mesurer les discriminations selon l'apparence : une analyse des contrôles d'identité à Paris », Population, vol. 67, nº 3, pp. 42351.

Jugnot S. (2019) «Les inégalités se mesurent, les discriminations se constatent », La Revue de l'Ires, vol. 98, ${ }^{\circ}$ 2, pp. 3-28.

L'Horty Y., Bunel M., Mbaye S., Petit P. et du Parquet L. (2019), « Discriminations dans l'accès à la banque et à l'assurance », Les enseignements de trois testings. Revue d'économie politique, $\mathrm{n}^{\circ} 1(1)$, pp. 49-78.

Leclerc O. (2013), «Égalité des personnes et modes de preuve. À propos des usages du raisonnement statistique dans la preuve des discriminations ", in Le droit social, l'égalité et les discriminations (Borenfreund G. et Vacarie I., dir.), Dalloz, pp. 77-94.

Martin O. (2020), L'empire des chiffres : Sociologie de la quantification, Armand Colin.

Porter M. T. (1996), Trust in numbers : the pursuit of objectivity in science and public life, Princeton University Press.

Petit P., Bunel M., et L'Horty Y. (2020), « Les discriminations à l'embauche dans la sphère publique : effets respectifs de l'adresse et de l'origine », Revue économique, vol. 71, $\mathrm{n}^{\circ} 1$, pp. 31-56.

Primon J.-L. (2011) « La perception des discriminations au filtre des enquêtes statistiques », Agora débats/jeunesses, vol. 57, $\mathrm{n}^{\circ} 1$, pp. 121-134.

Primon J-L. (2010). «La statistique est-elle indispensable ? Un débat français », Migrations Société, vol. 131, n 5, pp. 117-132.

Simon P. (2020) « Compter pour combattre », in Racismes de France (Slaouti O., dir.), La Découverte, pp. 366378.

Simon P. et Lesné M. (2012), « La mesure des discriminations dans l'enquête "Trajectoires et Origines" », Ined, Collection Documents de travail, $\mathrm{n}^{\circ} 184,32 \mathrm{p}$.

Spire A., et Merllié D. (1999), « La question des origines dans les statistiques en France. Les enjeux d'une controverse », Le Mouvement Social, $\mathrm{n}^{\circ}$ 188, pp. 119-130.

Stavo-Debauge J. (2003), «"Tu ne catégoriseras point !” ", Carnets de Bord, vol. 6.

Stavo-Debauge J. (2003), « Prendre position contre l'usage de catégories "ethniques" dans la statistique publique. Le sens commun constructiviste, une manière de se figurer un danger politique », in Historicité de l'action publique (Laborier P., Trom D., dir.), PUF, pp. 293-327.

Supiot A. (2015), La gouvernance par les nombres, Fayard. 1998, dossier " La variable "ethnie" comme catégorie statistique »(dossier), Population, vol. 53, $\mathrm{n}^{\circ} 3$, pp. 537-630. 